\title{
RESEARCH
}

Open Access

\section{The landscape of transposable elements and satellite DNAs in the genome of a dioecious plant spinach (Spinacia oleracea L.)}

Shu-Fen Li', Yu-Jiao Guo ${ }^{1}$, Jia-Rong Li', Dong-Xu Zhang ${ }^{2}$, Bing-Xiao Wang ${ }^{1}$, Ning Li ${ }^{1}$, Chuan-Liang Deng ${ }^{1 *}$ and Wu-Jun Gao ${ }^{1 *}$

\begin{abstract}
Background: Repetitive sequences, including transposable elements (TEs) and satellite DNAs, occupy a considerable portion of plant genomes. Analysis of the repeat fraction benefits the understanding of genome structure and evolution. Spinach (Spinacia oleracea L.), an important vegetable crop, is also a model dioecious plant species for studying sex determination and sex chromosome evolution. However, the repetitive sequences of the spinach genome have not been fully investigated.

Results: We extensively analyzed the repetitive components of draft spinach genome, especially TEs and satellites, by different strategies. A total of 16,002 full-length TEs were identified. Among the most abundant long terminal repeat (LTR) retrotransposons (REs), Copia elements were overrepresented compared with Gypsy ones. Angela was the most dominating Copia lineage; Ogre/Tat was the most abundant Gypsy lineage. The mean insertion age of LTR-REs was 1.42 million years; approximately $83.7 \%$ of these elements were retrotransposed during the last two million years. RepeatMasker totally masked about $64.05 \%$ of the spinach genome, with LTR-REs, non-LTR-REs, and DNA transposons occupying 49.2, 2.4, and 5.6\%, respectively. Fluorescence in situ hybridization (FISH) analysis showed that most LTR-REs dispersed all over the chromosomes, by contrast, elements of CRM lineage were distributed at the centromeric region of all chromosomes. In addition, Ogre/Tat lineage mainly accumulated on sex chromosomes, and satellites Spsat2 and Spsat3 were exclusively located at the telomeric region of the short arm of sex chromosomes.

Conclusions: We reliably annotated the TE fraction of the draft genome of spinach. FISH analysis indicates that Ogre/Tat lineage and the sex chromosome-specific satellites DNAs might participate in sex chromosome formation and evolution. Based on FISH signals of microsatellites, together with 45S rDNA, a fine karyotype of spinach was established. This study improves our knowledge of repetitive sequence organization in spinach genome and aids in accurate spinach karyotype construction.
\end{abstract}

Keywords: Repetitive sequence, Satellite DNA, Sex chromosome, Spinach, Transposable elements

\footnotetext{
*Correspondence: dcl75@163.com; gaowujun@htu.cn

'College of Life Sciences, Henan Normal University, Xinxiang 453007, China

Full list of author information is available at the end of the article
}

(c) The Author(s). 2019 Open Access This article is distributed under the terms of the Creative Commons Attribution 4.0 International License (http://creativecommons.org/licenses/by/4.0/), which permits unrestricted use, distribution, and reproduction in any medium, provided you give appropriate credit to the original author(s) and the source, provide a link to the Creative Commons license, and indicate if changes were made. The Creative Commons Public Domain Dedication waiver (http://creativecommons.org/publicdomain/zero/1.0/) applies to the data made available in this article, unless otherwise stated. 


\section{Background}

A substantial fraction of plant genomes is occupied by repetitive DNA, which mainly includes transposable elements (TEs) and satellite DNAs. TEs are DNA fragments that have the ability to move from one part of a genome to another, often accounting for a large proportion of the plant genome. They are categorized into two distinct classes based on structural feature and transposition pattern. Class I elements are also known as retrotransposons (REs), which can transpose via an RNA intermediate and self-replicate when transposed. Class II elements, also called DNA transposons, can move by direct "cut-andpaste" mode. Given that REs can increase their copy numbers after being transposed, they are usually the most abundant repetitive elements, especially long terminal repeat (LTR) REs. For example, in maize, REs and LTR-REs constitute 75.6 and $70.1 \%$ of the genome, respectively, whereas DNA transposons occupy 8.6\% [1]. LTR-REs exhibit typical structural features, such as the presence of LTRs at both ends, promoter and RNA processing signals, and flanking target site duplications (TSD) [2]. Near the inner $5^{\prime}$ LTR boundary and the inner 3 ' boundary are the primer-binding site (PBS) motif and polypurine tract (PPT), which respectively provide the signals required for the minus and plus DNA strand synthesis. The internal region of REs is generally divided into two open reading frames: GAG and POL. GAG encodes for a structural protein that packages the transcript into a virus-like particle. POL codes a polyprotein with protease, integrase (INT), reverse transcriptase (RT), and RNAseH (RH), which are essential for the replication and integration of elements in target regions [3]. Satellite DNA, also known as tandem repeat, is another type of repetitive element widely distributed in plants. It consists of a large number of repeat units (50-1000 bp) that are organized in tandem arrays [4].

These repetitive sequences are recognized to play important roles in various processes, such as in genome evolution [5, 6], chromosomal rearrangement [7], gene creation and regulation $[8,9]$. In addition, TEs and satellite DNAs can participate in various processes of plant sex chromosome evolution; such processes include recombination suppression, diversification of sex chromosome structure and morphology, sex chromosome degeneration, and dosage compensation [10, 11]. Thus, identification and annotation of TEs and satellites of the genomes of dioecious plants will lay foundation for further investigation of sex chromosome evolution.

Spinach (Spinacia oleracea L.) is an annual or biennial dioecious herbaceous plant belonging to Spinacia genus of Chenopodioideae family. The spinach genome is approximately $989 \mathrm{Mbp}$, with $2 \mathrm{n}=2 \mathrm{x}=12$ chromosomes. As a dioecious species, the sex type of spinach is determined by $\mathrm{X}$ and $\mathrm{Y}$ chromosomes. The $\mathrm{X}$ and $\mathrm{Y}$ chromosomes are homomorphic, indicating an early evolutionary stage of sex chromosomes in spinach. Current cytological research have demonstrated sex chromosomes as the longest pair of chromosomes [12, 13]. Given that repetitive sequences exhibit important effects on genome structure and evolution, a comprehensive analysis of repetitive sequences of the spinach genome is beneficial for understanding of the genome structure and evolution of spinach, especially of its sex chromosomes. A draft spinach genome has recently been published [14]. Although the authors annotated the repetitive sequence fraction of the draft genome, they only used the results of LTRharvest for TE identification and further annotation. A comparative study showed that LTRharvest without other software and methods for verification yields considerably high level of false positive ratio [15]. Thus, understanding of the repetitive sequences of spinach still needs further comprehensive analysis. Furthermore, the chromosome location, phylogenetic analysis, and evolution patterns of repetitive sequences of spinach genome remain to be studied.

Accurate identification and annotation of TE fraction in whole genome sequences are challenging tasks owing to the significant diversity of TEs [15]. Currently, a number of different methods and tools have been developed for detecting TEs in assembled genomes. Three strategies are commonly used: homology-based, signature-based, and de novo approaches $[15,16]$. Signature-based tools rely on the typical structure of a particular TE type and can detect individual full-length TEs, benefitting the investigation of the TE structure, variation, and evolution $[17,18]$. For comprehensive and reliable annotation of a given genome, the adoption of combined approaches with downstream verification has been shown to be the best strategy $[19,20]$.

In this study, based on the recently published draft spinach genome, we used different methods to identify and annotate the repetitive sequence fraction of the genome, with focus on TEs and satellite DNAs. We first used signature-based methods to identify full-length TEs and extensively analyzed the phylogeny, distribution, and insertion time of LTR-REs. Combined methods, including homology-based, signature-based, and de novo approaches, were then performed for annotating the TE fraction of the whole genome. We also analyzed the location pattern of different groups of TEs and satellite DNA using fluorescence in situ hybridization (FISH). This study can provide useful information for understanding the spinach genome structure with respect to TEs and satellites.

\section{Results}

Identification and annotation of full-length TEs

A total of 16,002 full-length TEs were detected in spinach draft genome using signature-based strategies. This 
dataset included 11,640 LTR-REs, 1020 non-LTR-REs, and 3342 DNA transposons. The full-length TEs totally comprised $125,231,331 \mathrm{bp}$, accounting for $12.57 \%$ of the draft spinach genome.

\section{LTR-REs}

LTR retroelements were identified using LTRharvest, and internal sequences were annotated using LTRdigest. First, LTRharvest predicted the presence of 17,734 sequences harboring two relatively intact LTRs and flanking TSDs. The $5^{\prime}$ and 3' ends of both LTRs were flanked by TG and CA. After LTRdigest analyses, we identified 11,640 putative full-length LTR-REs with PPT, or PBS sites, or at least one typical protein domain (Additional file 1). For 3290 and 2173 elements, the putative PBS and PPT were identified, respectively. A total of 4048 elements showed all typical protein domains of LTR-REs, but only 137 elements showed all the putative PBS, PPT, and at least one typical protein domain. The isolated LTR-REs covered a total of $110,438,058 \mathrm{bp}$, accounting for $11.09 \%$ of the whole genome. The LTR-REs ranged from $1191 \mathrm{bp}$ to $22,984 \mathrm{bp}$ in size. The mean length was $9787 \mathrm{bp}$, with a standard deviation of $4625 \mathrm{bp}$. The recorded putative LTRs showed a mean length of $689 \mathrm{bp}$, with large length variability (standard deviation $=549 \mathrm{bp}$ ).

The LTR-REs were classified into Copia or Gypsy superfamilies based on the order of the POL protein domains and on similarity searches against different public RE databases. The results showed that 5303 elements (45.6\%) belonged to Copia superfamily, whereas 3709 REs (31.9\%) were designated as Gypsy elements. There were still 2628 elements were classified as unknown because they lacked distinct protein-coding sequences sufficient for classification (Table 1).

The identified full-length REs were further analyzed for the presence of five typical RE protein domains (retrotranscriptase, RH, INT, protease, and GAG). Based on the similarity to lineage-specific RE protein domains, the Copia elements were subdivided into seven lineages, whereas the Gypsy elements belonged to six lineages (Fig. 1). The remaining 64 Copia and 48 Gypsy members were defined as unknown owing to the absence of sufficient similarity to known lineage-specific RE protein domains.

Among Copia elements, Angela lineage was predominant, accounting for more than $70 \%$ the full-length Copia elements, followed by Ale/Retrofit, SIRE, TAR, and Tork. Bianca and Ivana elements were the rarest, together

Table 1 Identification of full-length TEs in spinach genome

\begin{tabular}{|c|c|c|c|c|c|}
\hline Class & Order & Superfamily & No. & Total length (bp) & Percentage of genome (\%) \\
\hline \multirow[t]{12}{*}{ Retrotransposons } & \multirow[t]{3}{*}{ LTR } & Copia & 5303 & $51,884,973$ & 5.21 \\
\hline & & Gypsy & 3709 & $40,349,864$ & 4.05 \\
\hline & & Unclassifed & 2628 & $18,203,221$ & 1.83 \\
\hline & \multirow[t]{8}{*}{ LINE } & CRE & 642 & $2,981,624$ & 0.30 \\
\hline & & RTE & 149 & 824,520 & 0.08 \\
\hline & & 1 & 65 & 400,921 & 0.04 \\
\hline & & Tad1 & 46 & 287,739 & 0.03 \\
\hline & & R1 & 30 & 149,274 & 0.15 \\
\hline & & Rex & 25 & 150,849 & 0.02 \\
\hline & & R2 & 22 & 90,843 & 0.01 \\
\hline & & others & 41 & 227,457 & 0.02 \\
\hline & subtotal & & 12,660 & $115,551,285$ & 11.60 \\
\hline \multirow[t]{9}{*}{ DNA transposons } & \multirow[t]{6}{*}{$\mathrm{TIR}$} & Tc1-Mariner & 693 & 175,762 & 0.02 \\
\hline & & hAT & 397 & 144,071 & 0.01 \\
\hline & & CMC-EnSpm & 290 & 83,402 & 0.01 \\
\hline & & MULE-MUDR & 167 & 49,524 & 0.01 \\
\hline & & PIF & 26 & 9245 & 0.00 \\
\hline & & others & 27 & 7899 & 0.00 \\
\hline & MITE & & 445 & 160,590 & 0.02 \\
\hline & Helitron & & 1297 & $9,049,533$ & 0.91 \\
\hline & subtotal & & 3342 & $9,680,026$ & 0.97 \\
\hline Total & & & 16,002 & $125,231,331$ & 12.57 \\
\hline
\end{tabular}




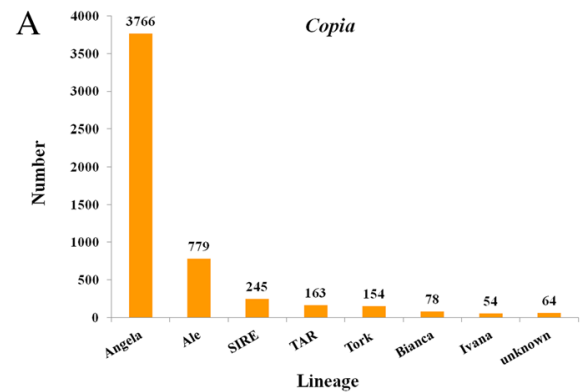

C

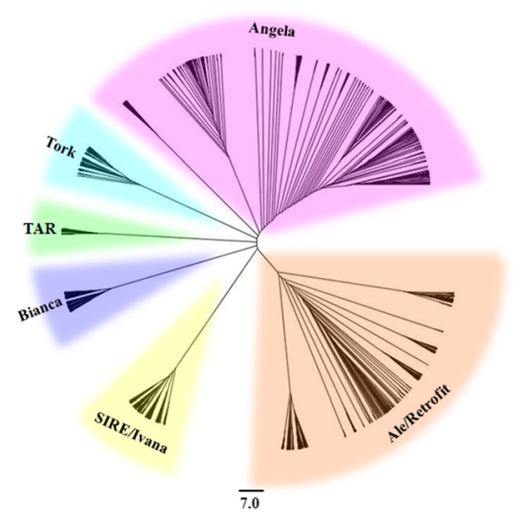

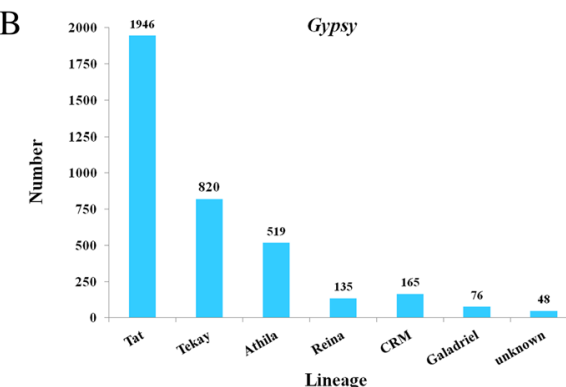

$\mathrm{D}$

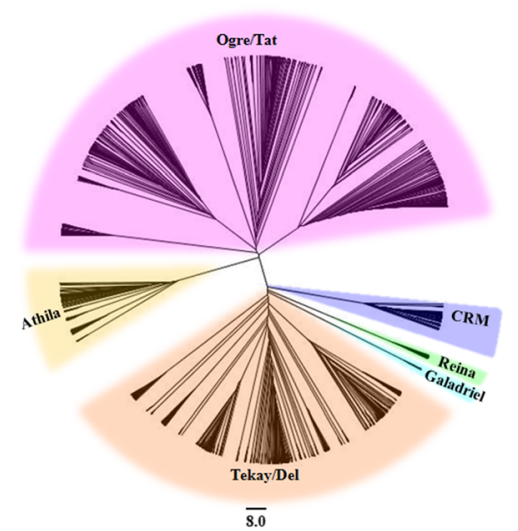

Fig. 1 Number and phylogenetic analysis of full-length REs identified in the spinach genome and were subdivided into superfamilies and lineages. The numbers of full-length retroelements in different lineages of Copia (a) and Gypsy (b) are shown. $\mathbf{c}$ and $\mathbf{d}$ represent the phylogenetic tree of RT domain protein sequences in different lineages of Copia and Gypsy, respectively. The scale bars indicate the average number of amino acid substitutions per site

representing 2.5\% of the Copia elements (Fig. 1). Analyses of Gypsy elements showed that Ogre/Tat, Tekay/Del, and Athila were the most common types of lineages. The other lineages, such as CRM, Reina, and Galadriel, were also identified but were less abundant, collectively accounting for $10.2 \%$ of the total Gypsy elements.

To determine the phylogenetic relationship of Copia and Gypsy REs, two phylogenetic trees were constructed based on the RT sequences of Copia and Gypsy REs. As shown in the evolutionary dendrograms (Fig. 1), among Copia REs, the Angela and Ale/Retrofit lineages showed higher variability, and could be further classified into three and two groups, respectively. SIRE and Ivana showed close relationship as they were clustered as one clade. The other lineages showed high homogeneity, as all sequences were clustered into a single clade for Tork, TAR, and Bianca (Fig. 1c). As for Gypsy REs, the most abundant Ogre/Tat lineage could be further classified into four groups, indicating the notable sequence diversity among this lineage. Ogre/Tat and Athila lineages both belonged to non-Chromovirus elements, and they showed close relationship and were grouped into one large clade. The other four superfamilies, Tekay/Del, Galadriel, Reina, and CRM, belonged to Chromovirus Gypsy REs and were clustered together (Fig. 1d).

\section{Non-LTR-REs}

A total of 1020 full-length non-LTR-REs were identified, occupying 5,113,227 bp and representing $0.5 \%$ of the spinach draft genome. The detected non-LTR REs all belonged to LINE order and included 12 superfamilies (Table 1). Among the different superfamilies, CRE elements were the most abundant, followed by RTE, I, and Tad1; the other superfamilies were rarely observed.

\section{DNA transposons}

The search for DNA transposons resulted in 1600 sequences classified as terminal inverted repeat (TIR) elements, 1297 as Helitron elements, and 445 as unknown miniature inverted repeat transposable elements (MITEs) (Table 1). Approximately $43.3 \%$ of the DNA transposons from the TIR order belonged to the superfamily Tc1-Mariner. Other identified elements belonged to hAT, CMC-EnSpm, MULE-MuDR, and PIF superfamilies. A total of 67 elements also belonged to other superfamilies, such as Dada, Maverick, and Kolobok (Table 1).

\section{Insertion time analysis of LTR-REs}

According to nucleotide substitution between two terminal LTRs of each LTR-REs, we estimated the insertion 
time of the identified LTR-REs. The putative mean age of analyzed LTR-REs is 1.42 million years (MY). Nearly $83.7 \%$ of them inserted in the last $2 \mathrm{MY}$, with a peak of activity was observed at $\sim 0.5-0.8 \mathrm{MY}$. In general, the Copia and Gypsy elements were younger than the unknown elements. The mean insertion ages of Copia, Gypsy, and unknown elements are 1.18, 1.05, and 1.55 MY, respectively (Fig. 2). The oldest element was an unknown element, with a putative insertion age of 19.6 MY. Among the $>10$ MY elements, 4, 1, and 10 belonged to Copia, Gypsy, and unknown elements, respectively. Analysis of insertion dates of the main Copia and Gypsy lineages showed that different lineages underwent retrotransposition in different time spans (Fig. 3). The youngest lineage was Reina, which belonged to the Gypsy lineage, with mean and median insertion ages of $0.70 \pm 0.07$ and $0.35 \mathrm{MY}$, respectively. By contrast, in a Copia lineage, Angela showed the oldest putative insertion date, with mean and median insertion ages of 1.31 \pm 0.02 and of $1.00 \mathrm{MY}$, respectively.

\section{Repeat composition of the spinach draft genome}

Based on combined strategies including de novo-, signature-, and homology-based methods, overall, TEs masked approximately $64.05 \%(637,890,846 \mathrm{bp})$ of the spinach draft genome. The LTR-REs, non-LTR-REs, and DNA transposons occupied 490, 23.9, and $55.5 \mathrm{Mb}$ DNA sequences, accounting for 49.2, 2.4, and 5.6\% of the genome, respectively. A total of $68.4 \mathrm{Mb} \mathrm{TE}$ sequences were unclassified, representing $6.9 \%$ of the genome (Fig. 4 a). Out of the most abundant TE element in LTR-REs, Copia and Gypsy represented approximately 24.2 and $18.9 \%$ of the genome, respectively (Fig. 4b).

\section{Chromosome localization analysis of LTR-REs}

We analyzed the chromosome distribution patterns of all the lineages of LTR-REs on spinach mitotic chromosomes. The results showed that elements from distinct evolutionary lineages exhibit different patterns of genomic distribution. Most of the lineage-based elements were dispersed over all of the chromosomes; these included four lineages of the Copia superfamily (Ale/Retrofit, SIRE, TAR, and Tork) and five lineages of the Gypsy superfamily (Tekay/ Del, Athila, Galadriel, and Reina) (Fig. 5). The other three lineages (Angela, Binaca, and Ogre/Tat) were mainly occupied the pericentromeric regions of all chromosomes, and the signals of Ogre/Tat on sex chromosomes were brighter and more intensive than on other autosomes (Fig. 5). CRM elements were predominantly located at the centromeric region of all chromosomes (Fig. 5). In addition, a probe derived from Ivana elements failed to give visible signals, most probably due to relatively low copy number.

\section{Satellite identification and chromosome location analysis}

We used TAREAN for identification of satellite DNAs using unassembled reads. A set of randomly selected 2,000,000 reads with average length of $301 \mathrm{bp}$, amounting to $0.6 \times$ spinach genome equivalent, was used for analysis. The results showed that in addition to rDNA,

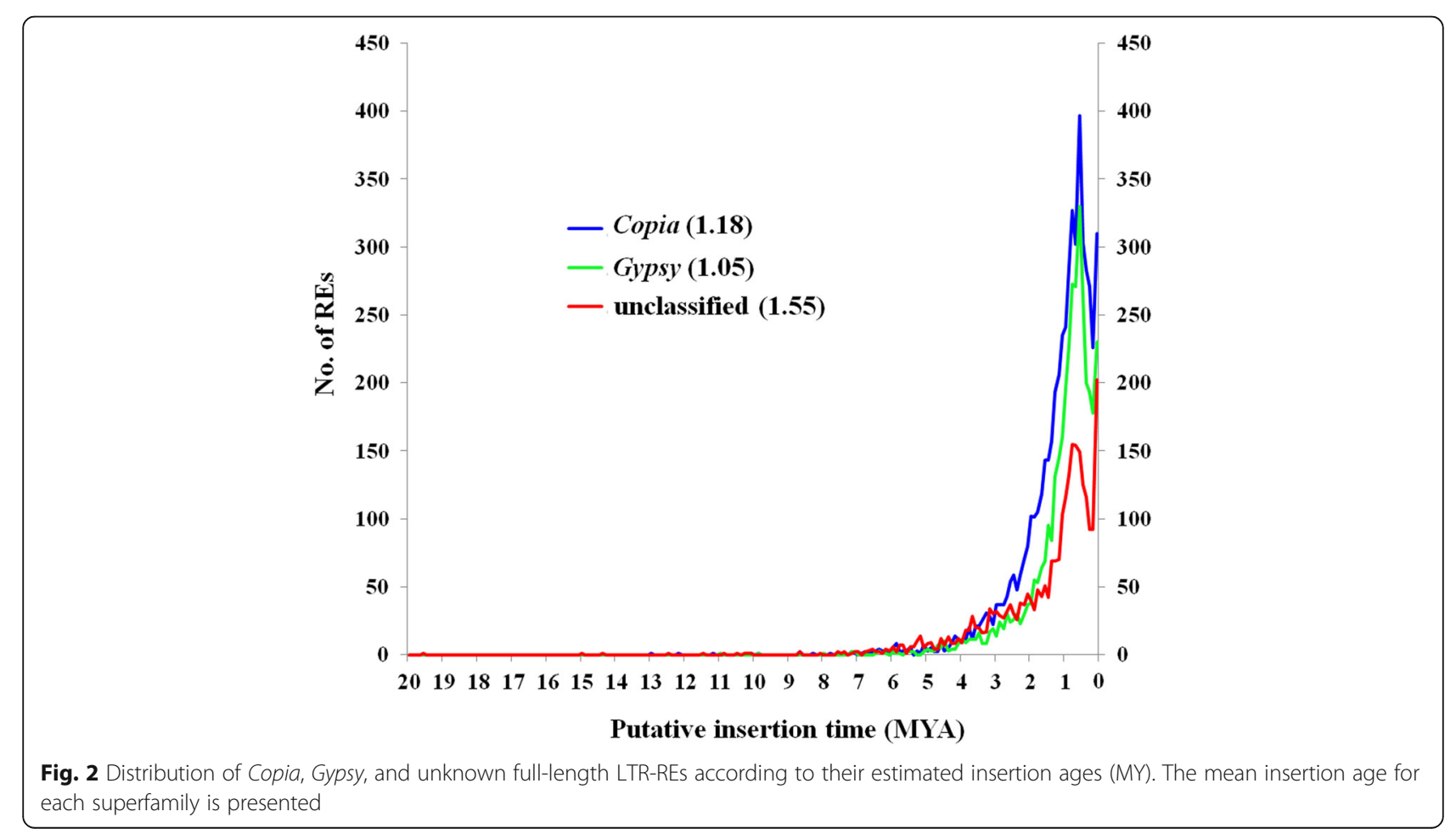




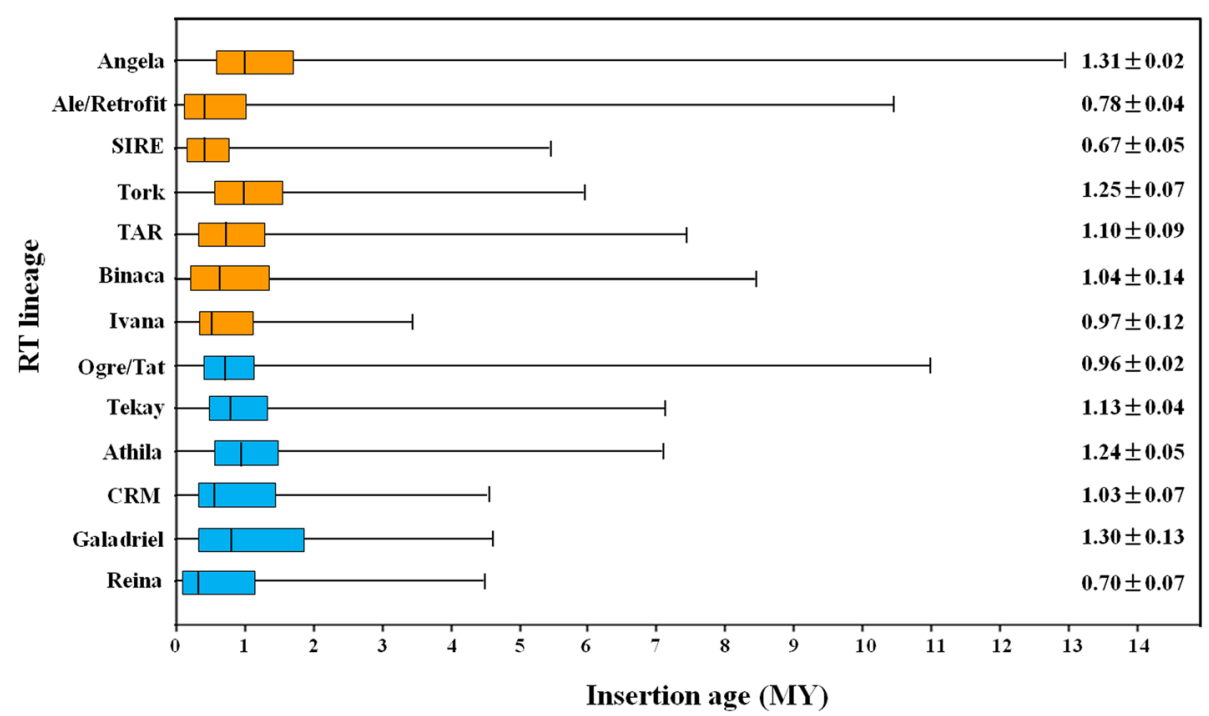

Fig. 3 Putative insertion ages of the main spinach LTR-RE lineages. The boxes represent 25-75\% of each LTR-RE lineage, whiskers represent the whole range of values, and vertical lines in the box represent the medians of distribution. For each lineage, the mean insertion age ( \pm SE) is reported

three clusters (CL51, CL162, and CL208) were identified as satellite DNAs. We designated them as Spsat1, Spsat2, and Spsat3, respectively. These clusters featured a star-like or circular graph topology (Additional file 2). The Spsat1 was estimated to make up $0.47 \%$ of the genome. The cluster showed $\sim 52 \mathrm{bp}$ monomers, and the monomers presented remarkably high similarity (Additional file 2). Searching GenBank revealed no similarities to other known sequences. FISH on mitotic chromosomes showed that the signals of Spsat1 concentrated on the telomeric regions of two pairs of chromosomes. In one pair of chromosomes, the telomeric region of the short arm showed

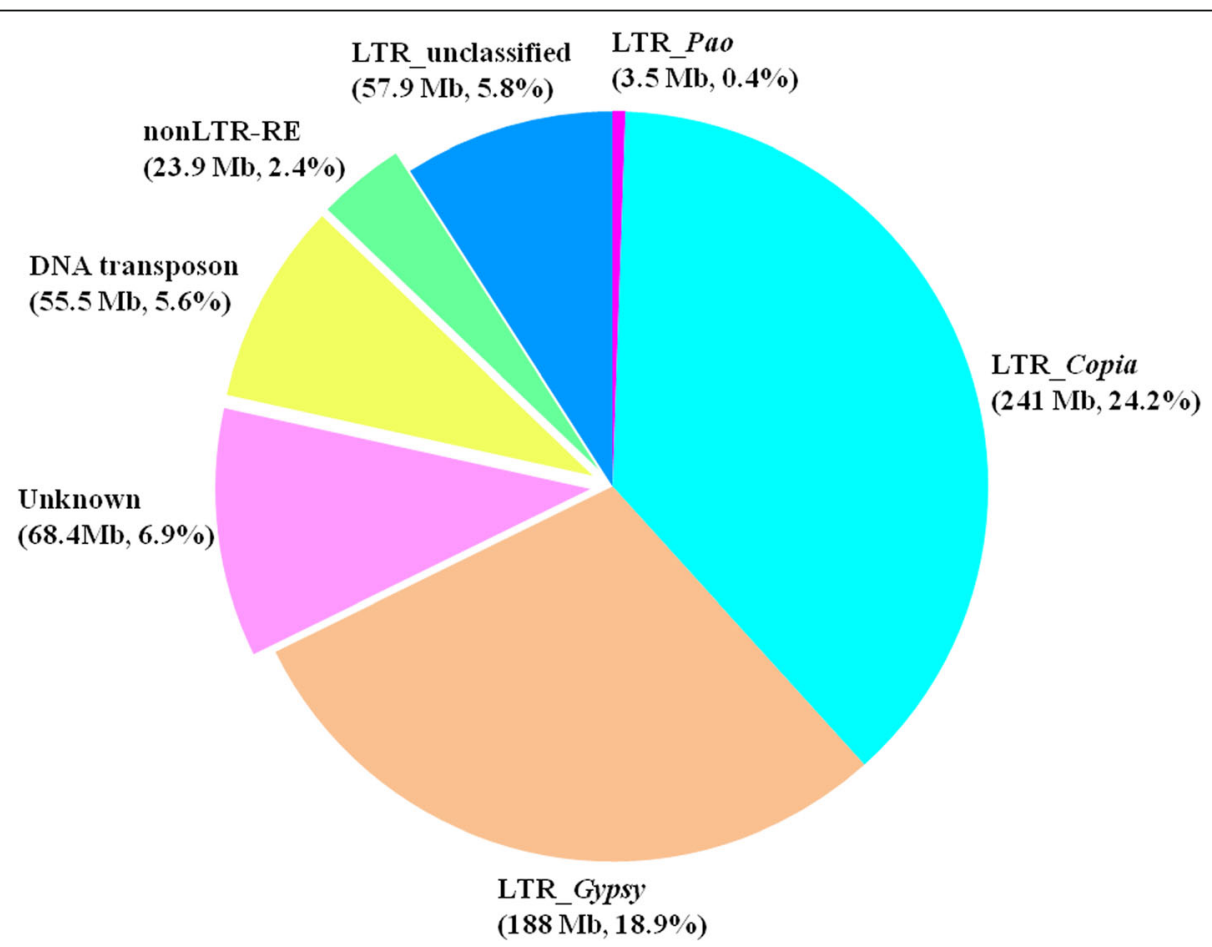

Fig. 4 TE annotation of spinach draft genome. Whole TE fraction, total length, and genome proportion of nonLTR-RE, DNA transposon, unknown elements, and LTR-RE subdivided into each superfamily are shown 


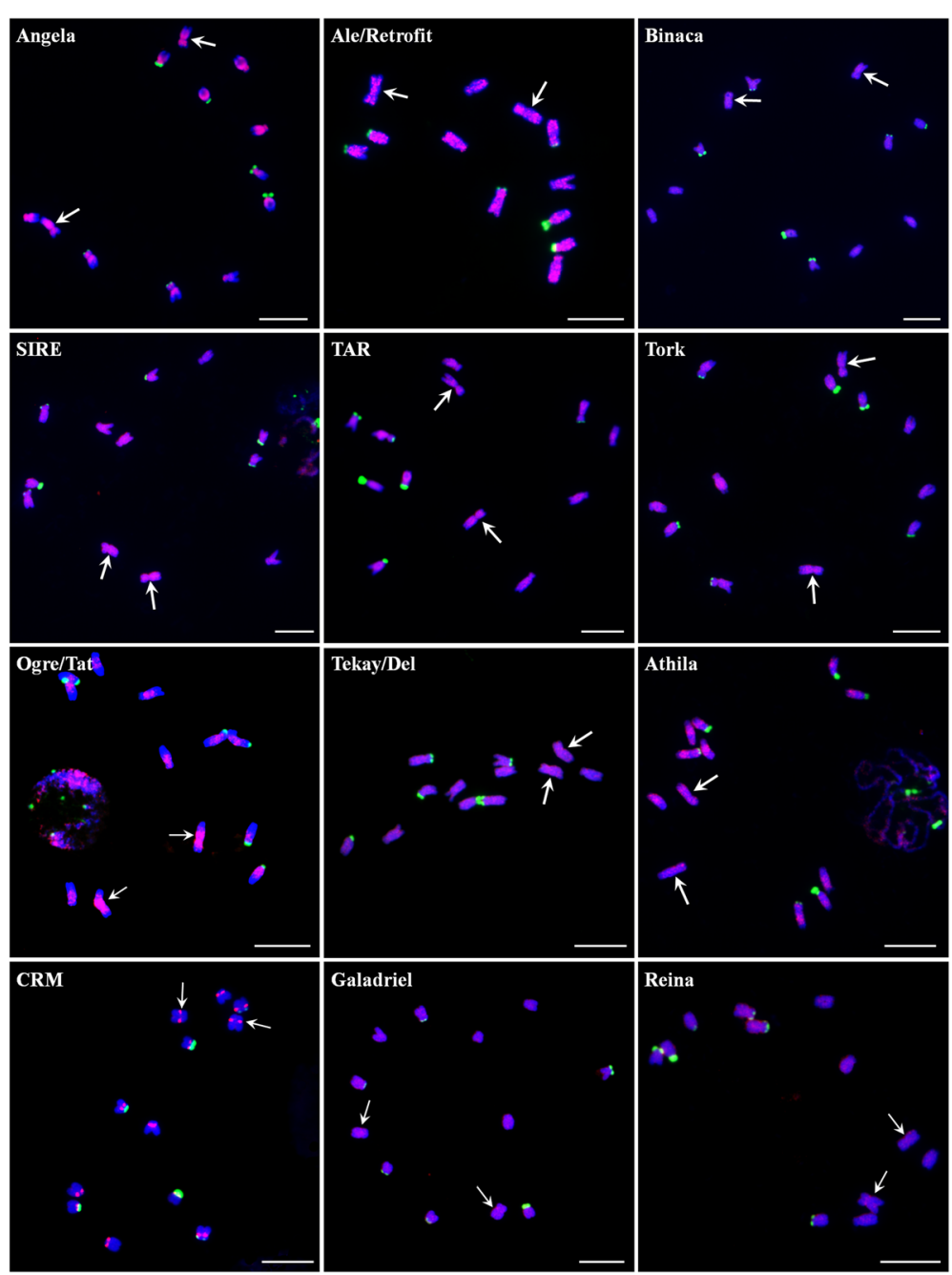

Fig. 5 Distribution patterns of different LTR-RE lineages on spinach chromosomes. The RT sequences of each lineage were labeled with Texas red (red signal), 455 rDNA was labeled with Chroma Tide Alexa Fluor 488 (green signal), and the chromosomes were counterstained with DAPI (blue). Arrows indicate the sex chromosomes with more brighter signals using probe of Ogre/Tat lineage RT sequence. Arrows indicate the sex

chromosomes. Bars $=10 \mu \mathrm{m}$

strong signals, whereas that of the long arm showed relatively weak signals. In another pair of chromosomes, signals were distributed on the telomeric region of the long arm (Fig. 6a). FISH on meiotic chromosomes showed that three signals, one large and two small, were detected on the pachytene chromosomes. In diakinesis period, two bivalent chromosomes, one with signals at both ends and one with signals at one end, were observed. In metaphase I, the strong signals on the short arm of one pair of chromosomes were directed poleward, whereas the other signals were at the middle of the bivalent chromosomes (Fig. 6b).

The other two satellites Spsat2 and Spsat3 showed $\sim 365$ and $\sim 325$ bp monomers, respectively. Similar to Spsat1, the clusters were all unknown or spinach-specific sequences. Mitotic FISH revealed that these clusters were located at the telomeric regions of the short arm of one pair of chromosomes (Fig. 6a). According to a comparison of the chromosomes, this pair of chromosomes is the largest, that is, they are sex chromosomes. For meiotic FISH analyses, one clear signal near the end of one chromosome was detected in pachytene and diakinesis periods (Fig. 6b). In metaphase I, the signals on one pair of chromosomes were directed poleward (Fig. 6b), indicating the signals were closer to the centromeres of the chromosomes.

To precisely identify each pair of homologous chromosomes and obtain detailed molecular karyotype of spinach, we performed sequential FISH using satellite DNA sequences (Spsat2, Spsat1, and $45 \mathrm{~S}$ rDNA) as probes. Based on the signals of the three probes, an accurate karyotype of spinach was established (Fig. 7). The Spsat2 signals concentrated on the telomeric regions of the 


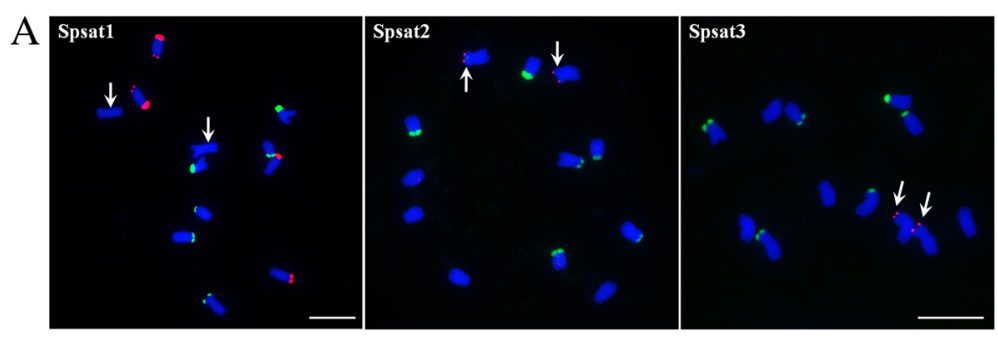

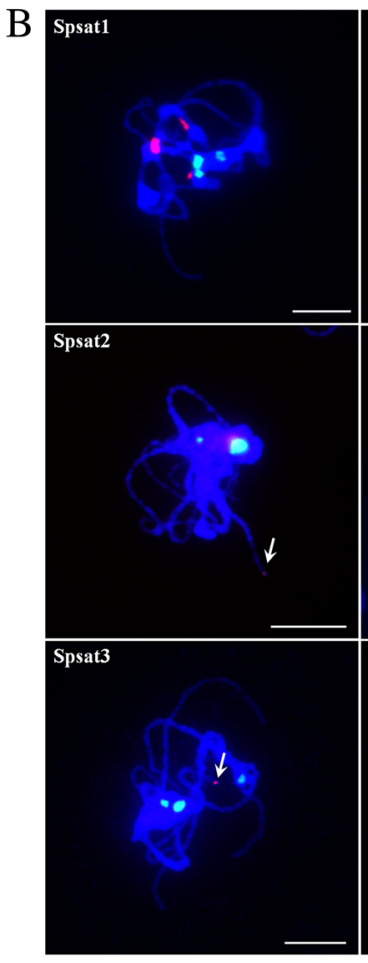

Pachytene

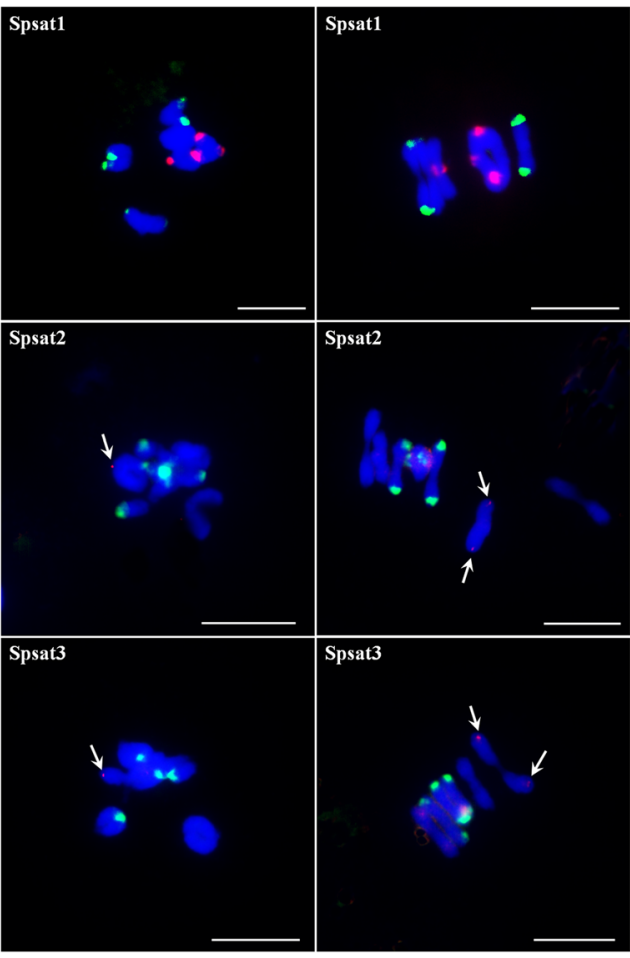

Diakinesis

Metaphase I

Fig. 6 FISH mapping of three satellites on both mitotic and meiotic chromosomes in spinach. a FISH analysis of Spsat1, Spsat2, and Spsat3 on mitotic chromosomes. Arrows indicate the sex chromosomes (Spsat1) or the signals on the sex chromosomes (Spsat2 and Spsat3); b FISH analysis of Spsat1, Spsat2, and Spsat3 on meiotic chromosomes; three typical phases: pachytene, diakinesis, and metaphase I are shown. Arrows indicate the signals of the satellite DNAs. The satellite DNAs were labeled with Texas red (red signal), 45S rDNA was labeled with Chroma Tide Alexa Fluor 488 (green signal), and the chromosomes were counterstained with DAPI (blue). Bars $=10 \mu \mathrm{m}$

short arm of chromosome 1 (sex chromosome). The Spsat1 signals were located at the telomeric regions of both the telomeric regions of chromosome 3 and the long arm of chromosome 4. The $45 \mathrm{~S}$ rDNA was mainly distributed on the telomeric regions of the three other pairs of chromosomes, i. e. chromosomes 2, 5 , and 6.

\section{Discussion}

\section{TE annotation of spinach draft genome}

Using combined annotation strategies, TEs represent $64.05 \%$ of the spinach draft genome, and this value is slightly lower than that of a previous report on the same genome [14]. Compared with that previous study, we used more TE detection software with more stringent analyses and could thus obtain more reliable results. The proportion of TE fraction of different plant genomes presented significant variation. For example, TEs represent less than 20\% of the Arabidopsis genome [21] but occupy more than $85 \%$ of the wheat genome [18]. In general, the quantity of TEs, especially LTR-REs, correlates well with the genome size of higher plants [7]. Spinach possesses a medium-large genome with $996 \mathrm{Mb}$, and the TE fraction proportion is generally in line with the trend. Among the TEs, REs are usually far more abundant than transposons, whereas LTR-REs are predominant TE elements in plant genomes [7]. As expected, REs represented $49.2 \%$ of the spinach genome, whereas DNA transposons occupied a small proportion (5.6\%). The prevalence of REs, particularly LTR-REs, was mainly due to their intrinsic proliferation characteristics [22]. Among LTR-REs, Copia elements 


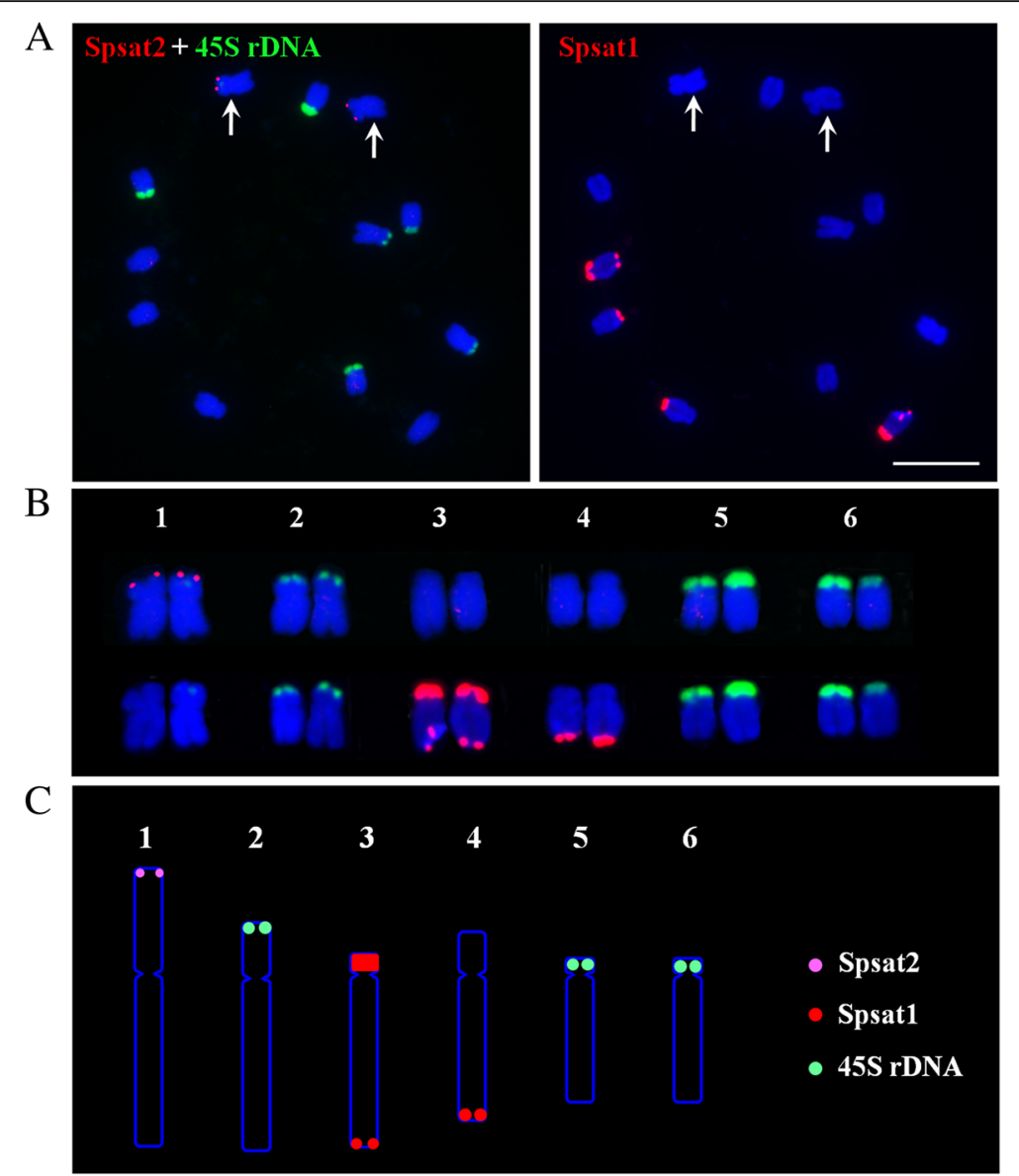

Fig. 7 Karyotype and ideograph for spinach mitotic metaphase chromosomes. a FISH with Spsat2 (red) and 45S rDNA (green), and the same spread was probed with the probe of Spsat1 (red). $\mathbf{b}$ Karyotype analysis of spinach based on the size and FISH signal pattern. $\mathbf{c}$ Ideogram shows the positions of Spsat2 (purple), Spsat1 (red), and 45S rDNA (green). Bars $=10 \mu \mathrm{m}$

were more abundant than Gypsy elements in the spinach genome. This result disagrees with previous findings, which showed the prevalence of Gypsy elements in spinach genome [15]. This result may be caused by the different annotation methods used. A large difference in the proportion of Copia and Gypsy elements was observed among different plant genomes. Different ratios between Gypsy and Copia element frequencies were reported, with values ranging from 10:1 in Gossypium arboreum [23] to 1:4 in Elaeis guineensis [24]. Among the 86 plant genomes we collected in a previous paper [7], Gypsy elements predominate in 64 genomes, whereas the other 22 are Copia-biased. This observation indicates that different genomes show unique retroelement expansion patterns, which are mainly due to different evolutionary processes within each plant species [25].

\section{Diversity and dynamics of full-length LTR-retroelements in the spinach genome}

Given that LTR-retroelements are usually far more abundant than other types of TEs, and identification of full-length elements benefits the investigation of structural variability, diversity, and phylogenetic evolution of TEs in spinach genome, we analyzed full-length LTR-REs in detail. In this study, the most abundant full-length LTR-REs belonged to Copia, followed by Gypsy, and the remainder elements were unknown. Among the lineages, Angela and Ale Copia REs were more redundant than the other Copia lineages, whereas Ogre/Tat was the most redundant Gypsy lineage. These results suggest that the proliferation of certain lineages, such as Angela and Ogre/ Tat, contributes significantly to spinach genome evolution. The prevalence of particular repeat lineages or families differs dramatically among different plant species. In numerous cases, a limited number of repetitive types are highly amplified. For example, five families of LTR-REs represent approximately $80 \%$ of the maize RE repertoire [1, 26], and a single Ty3-Gypsy-like RE accounts for approximately $38 \%$ of the genome of Vicia pannonica [27]. The mechanisms behind the proliferation of several RE families or lineages are poorly understood, and the most accepted explanation is that these families or lineages lost 
their silencing cellular mechanisms of the host genome $[28,29]$.

The LTR-REs showed considerable structural diversity. During retrotransposition, the active LTR-REs should exhibit all the elements that facilitate retrotransposition, including those of terminal LTRs, PBS and PPT sites, and all the necessary protein domains. However, as selective pressure usually features no or slight influence on TEs, TEs usually undergo rapid evolution process, such as truncations, nested insertions, and mutations [30]. These variationss result in the structural diversity of TEs. We discovered that only 145 full-length REs possessed all the required elements for retrotransposition, indicating that most of the REs in spinach are inactive. The inactivation of TEs is a protection mechanism to genome stability because a high level of TE activity is widely believed to induce genome instability, which is harmful for the genome [31].

The two LTRs of a retroelement were identical at the time of insertion and subsequently diverge due to random mutations, thus facilitating the estimation of insertion time of REs [26]. The mean RE insertion date was $1.42 \mathrm{MY}$, and the majority of retrotransposons were accumulated within the last two million years, indicating the very recent and probably still occurring RE burst. The recent RE proliferation events might exhibit important influence on spinach genome structure and evolution. A similar time course of RE amplification wave was reported in other herbaceous species, such as rice, wheat, Solanum lycopersicum, and Arabidopsis [25, 32, 33]. In spinach, the mean insertion data of Copia full-length REs was lower than that of Gypsy REs. However, the insertion date profiles indicate that Copia and Gypsy REs have experienced similar time courses.

\section{Repetitive sequences and sex chromosome evolution of spinach}

FISH analysis showed that Ogre/Tat lineage accumulated more in the sex chromosomes than in autosomes. In addition, two satellite DNAs were exclusively located in sex chromosomes. These results suggest that repetitive sequences, including TEs and satellites, accumulated more in sex chromosomes than in autosomes. Several studies have demonstrated that repetitive sequences accumulate in sex chromosomes in both plants and animals $[10,34]$. For example, in papaya, repetitive sequences occupy $79.2 \%$ of MSY and $67.2 \%$ of the X chromosome counterpart, whereas the ratio of repetitive sequences in the entire genome is $51 \%[35,36]$. In another dioecious plant Rumex acetosa, a number of satellites are located in sex chromosomes or Y chromosomes only [37]. The TEs and TE-derived repetitive sequences are believed to participate in nearly all the main evolutionary steps of sex chromosome evolution, such as recombination suppression, heterochromatization, chromosome morphology and structure alteration, and Y chromosome degeneration [10]. In addition, TEs and related repetitive sequences may regulate sex determination and differentiation of plants. For example, in the monoecious plant melon, one TE is inserted into transcription factor $C m W I P 1$, leading to the methylation of the flanking transcription factor sequence of the TE and causing the development of unisexual male flowers [8]. Spinach possesses a pair of young homomorphic sex chromosomes (X and Y). The sex chromosome-biased TEs and sex chromosome-specific satellites may play a role in sex chromosome formation and evolution in spinach.

By contrast, it should be noted that several repetitive seqeunces are ubiquitously distributed in autosomes but are absent in sex chromosomes [38-40]. For example, one family of Ogre/Tat lineage is nearly absent in the $\mathrm{Y}$ chromosome but is distributed widely in autosomes and $\mathrm{X}$ chromosome in Silene latifolia [39]. We also found that a satellite DNA is distributed at the telomeric regions of autosomes and X chromosome, but not in Y chromosome in Humulus scandens (our unpublished results). Thus, the relationship between repetitive sequences and the evolution of plant sex chromosomes is complicated. Based on the current limited reports [10, 38-40], it seems that the accumulation or depletion of which type of repetitive sequences is species-specific, which is consistent with the fact that sex chromosomes have evolved many times indepedently in plants [41].

Sex chromosomes in most dioecious plants evolved much more recently compared with most of animal sex chromosomes. The evolution time of human sex chromosome is approximately 240-300 MY, whereas most of the plant sex chromosomes emerged within the past 25 MY [42, 43]. For instance, sex chromosomes of Silene latifolia and Coccinia indica evolved less than 10 MY [44, 45]. The homomorphic sex chromosomes of Carica papaya, Fragaria viginiana, and Rumex hastatulus evolved even more recently, with evolution times of 2.5, 1, and 0.6 MY, respectively [46-48] (Fig. 8). Presently, no study reported the evolution time of sex chromosomes in spinach. However, given that $\mathrm{X}$ and $\mathrm{Y}$ chromosomes are homomorphic, and the $\mathrm{YY}$ individual can survive, we posit that the sex chromosomes of spinach must be young. Studies have reported that plant REs mainly evolved within the last 1-12 MY $[49,50]$. Therefore, the majority of the sex chromosomes of dioecious plants emerged after plant RE evolution. Thus, we propose that sex chromosome origin and evolution may be closely related to the burst of repetitive sequences, mainly REs. In this study, the rapid amplification of REs occurred within the last two million years, and this RE proliferation event may be involved in the origin and evolution of spinach sex chromosomes. In the future, 


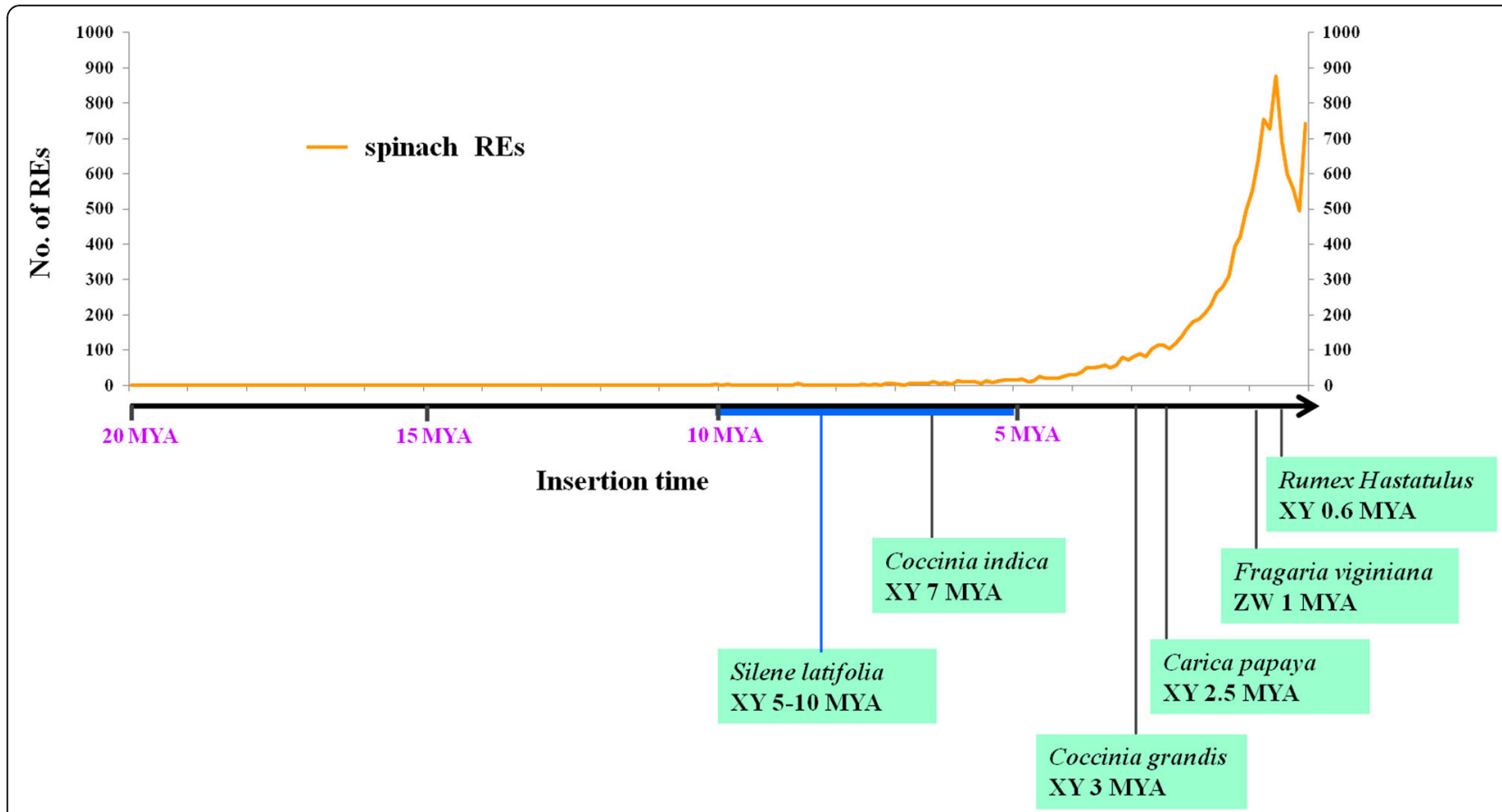

Fig. 8 Evolutionary age of the reported sex chromosomes of dioecious plants and insertion age of LTR-REs in spinach

with the complete assembly of sex chromosomes, we can investigate the relationship of repetitive sequences and sex chromosome formation and evolution in detail.

\section{Molecular karyotypic analyses of spinach}

Although spinach is a popular vegetable and a model dioecious plant for studying sex chromosome evolution and sex determination mechanism, few cytogenetic studies were carried out, and detailed molecular karyotype analyses that reliably distinguish each chromosome of spinach have not been conducted. Chromosome identification is essential for cytological analyses and subsequent studies in genomics, taxonomy, and evolution of sex chromosomes, establishing a bridge between visible landmarks and genetic or physical map features. The satellites identified in this study provide good markers for karyotyping analysis. Based on satellite identification and FISH analysis, we obtained three useful satellite DNAs (Spsat1, Spsat2, and Spsat3) which can be used as cytogenetic markers. Together with rDNAs, one or two signals were detected on each chromosome. We can now easily identify all six somatic metaphase chromosomes by the position of FISH signals. Based on sequential FISH, an integrated metaphase chromosome karyotype was established, providing a valuable basis for future cytogenetic and genomic studies.

\section{Conclusions}

In conclusion, this study presents a comprehensive description of the repetitive sequence organization of spinach, an important vegetable and dioecious plant species. We reliably annotated the TE portion of the spinach draft genome based on combined strategies, with LTR-REs representing $49.2 \%$ of the spinach genome. The fulllength LTR-RE elements allowed us to investigate the structural variation, phylogenetic relationship, and insertion dynamics of the most abundant genome fraction. Our TE database will serve as resource in future studies aimed at assessing the possible contribution of TEs to genome structure and evolution. FISH analysis showed that one lineage of LTR-RE and two satellites accumulated on sex chromosomes, suggesting that repetitive sequences may play important roles in spinach sex chromosome evolution. The satellites identified in this study provide good markers for future cytogenetic analysis of spinach.

\section{Materials and methods}

\section{Plant materials}

The spinach variety Daye (II 9A0002) was used in this study. Seeds were sown and grown in a garden field of Henan Normal University under natural conditions. Total genomic DNA was extracted from young leaves using traditional cetyl trimethylammonium bromide method.

\section{Identification of TEs}

The spinach draft genome was downloaded from http://www.spinachbase.org/?q=download. We used LTRharvest [51] to identify LTR-REs, and the parameters included the following: LTR size of $100-5000 \mathrm{bp}$, 
minimum distance between LTRs of $1000 \mathrm{bp}$, maximum distance between LTRs of 20,000 bp, flanking by dinucleotides TG and CA at $5^{\prime}$ and $3^{\prime}$ of each LTR, similarity of $60 \%$, TSD of $4-8 \mathrm{bp}$, and other default parameters. The internal features of the identified putative LTR retroelements were annotated by using LTRdigest program [52]. A database of tRNAs using tRNAscan-SE (version 1.3.1) [53] was constructed to predict the location of PBS, and the hidden Markov model profiles were downloaded from the Gypsy [54] (http://gydb.org/index.php/Main_Page) and Pfam databases [55] (http://pfam.xfam.org 31.0). The putative full-length LTR-REs with typical LTR-RE features, that is, with a putative $15-18$ nt PBS or a $10-30$ nt PPT upstream of the $5^{\prime}$ end of the $3^{\prime}$ LTR or possessing one of the typical RE protein domains (GAG, protease, RT, RH, and INT), were filtered for further analysis; the remaining LTR-REs were discarded. The identified LTR-REs were first classified into Copia or Gypsy superfamilies according to the order of the RT and INT domains, that is, the Copia candidates showed the INT-RT order, whereas the Gypsy ones showed the RT-INT order. The protein domains of the identified full-length LTR-REs belonging to Gypsy or Copia superfamiles were then extracted using RepeatExplorer [56] and were used as reference datasets for further BLASTX queries to classify previously unclassified elements. Finally, the still unclassified elements were further classified by BLASTN and BLASTX searches against Viridiplantae TE database retrieved from Repbase (http:// www.girinst.org/repbase, 23.07) and public sequence databases (non-redundant nucleotide and protein National Center for Biotechnology Information (NCBI) databases). We used $\mathrm{E}$ value thresholds of $\mathrm{E}<10^{-10}$ and $\mathrm{E}<10^{-15}$ for BLASTN and BLASTX, respectively.

For non-LTR-REs, MGEScan-nonLTR program [57] was used with default parameters. The results of this program were well-classified superfamilies of non-LTRREs. To detect Helitron DNA transposons, HelitronScanner software [58] was used. We used the parameters of headscore 8 and tailscore 10 to obtain reliable results. Small non-autonomous DNA transposon elements were identified by using MUSTv2 program [59] and MITE_Hunter [60]. The results obtained by these software were combined and annotated by using RepeatClassifier program (v1.0.10), which is a part of the RepeatModeler package, and then finally checked manually.

\section{Phylogeny and classification of LTR-REs}

The LTR-REs were classified into known lineages and clades according to the phylogenetic relationships of their RT protein domains, which were extracted from full-length REs using the RepeatExplorer platform. After removing redundant sequences using CD-hit [61], multiple alignment of the consensus RT domain sequences was carried out using MUSCLE [62]. The alignment was used to construct phylogenetic trees by using FastTree [63]. The trees were drawn and further edited using FigTree software.

\section{Insertion time estimation of LTR-REs}

The 5 ' - and 3 '-LTRs of each putative full-length LTR-REs were compared to estimate the insertion age of LTR-REs [64]. After the two LTRs of each RE were aligned by using program "Stretcher" (EMBOSS package) [65], the nucleotide distance between two LTRs were measured using the Kimura two-parameter method [66]. An average substitution rate $(\mathrm{r})$ of $1.3 \times 10^{-8}$ substitutions per synonymous site per year and insertion time $(\mathrm{T})$ formula $\mathrm{T}=\mathrm{k} / 2 \mathrm{r}$ were then employed to measure insertion time [32].

\section{TE annotation of the spinach genome}

TE annotation was performed by using RepeatMasker based on libraries generated by different strategies: de novo-based, signature-based, and homology-based methods. De novo identification of TEs was performed using RepeatModeler (http://www.repeatmasker.org/RepeatModeler, version 1.0.10). The consensus families generated by RepeatModeler (Additional file 3) were used as a custom library to mask the spinach genome by RepeatMasker (v4.0.7). The spinach genome was then masked using the library of previously classified TE sequences identified by signature-based methods (Additional file 4). The unmasked sequences were further analyzed by RepeatMasker using the filtered consensus family sequences as a custom library. Finally, the consensus TE sequences from Repbase were used as a library to mask the remaining unmasked sequences using RepeatMasker. The results of the above three steps were combined and analyzed.

\section{Identification of satellite DNAs}

A set of whole genome Illumina Miseq paired-end reads with average length of $301 \mathrm{bp}$ was downloaded from NCBI with Sequence Read Archive accession number of SRR4447192 [67]. After filtering using HTQC (v1.92.1) [68], a randomly selected dataset containing 2,000,000 reads, which represented $0.6 \times$ spinach genome, was used for graph-based clustering analysis using TAREAN online pipeline [69]. Clustering analysis was performed using a threshold of $90 \%$ similarity over at least $55 \%$ of the sequence length. Clusters containing satellite repeats were identified based on graph topology and software estimation results. The genome proportion of each putative satellite DNA cluster was calculated as the percentage of reads, which is the number of reads in each cluster divided by all the reads used in the graph-based clustering. 
The logo for the satellite sequence was generated by Web-logo [70].

\section{Preparation of probes for FISH}

The FISH probes used in this study were produced from two sets of data, that is, the RT domains of the lineages of LTR-REs and satellites. To investigate the distribution of major lineages of LTR-REs, we amplified RT domains of Copia and Gypsy REs using designed specific primer sets (Additional file 5). PCR products were checked by gel electrophoresis, and the desired bands were cleaved, cloned into pEASY-T1 vector (Transgene, Beijing, China), and transformed into competent Escherichia coli cells. The positive clones were screened and sequenced (Sangon Biotech, Shanghai, China) to verify the presence of specific RT domains in the clones. Clones with at least 90\% similarity to the corresponding reconstructed contigs were PCR-amplified and labeled with Texas-red-dCTP (PerkinElmer, Waltham, Massachusetts, USA) using nick translation method as described previously [71]. The monomers of satellites were synthesized with $5^{\prime}$-Texas-red modification (Invitrogen, Shanghai, China). For improved characterization of the chromosomes, 45S rDNA was labeled with Chroma Tide Alexa Fluor 488-5-dUTP (Invitrogen) for FISH.

\section{Chromosome preparation and FISH analysis}

Mitotic metaphase spreads were prepared from meristem root tip cells following previously used procedures [13] with minor modifications. Briefly, spinach seeds were cultured on moist papers in dishes at $25^{\circ} \mathrm{C}$. After 1-2 days, root tips with approximately $1 \mathrm{~cm}$ length were cut and pretreated with nitrous oxide gas for $2 \mathrm{~h}$. The root tips were then fixed in ice-cold $90 \%$ acetic acid for $10 \mathrm{~min}$ and stored in $70 \%$ ethanol at $-20^{\circ} \mathrm{C}$. The root tips were washed in ice-cold $1 \times$ citric buffer for $10 \mathrm{~min}$. The root sections with actively dividing region were excised and incubated in an enzyme mixture containing $1 \%$ pectolyase Y 23 (Yakult Pharmaceutical, Tokyo, Japan) and 2\% cellulose Onozula R10 (Yakult Pharmaceutical) for $2 \mathrm{~h}$ at $37^{\circ} \mathrm{C}$. After digestion, the root sections were washed in ice-cold TE and $100 \%$ ethanol twice in sequence. The root sections were fine-broken with a needle and vortexed at $4000 \mathrm{rpm}$ for $20 \mathrm{~s}$. The cells were collected by centrifugation and resuspended in $100 \%$ acetic acid to prepare a cell suspension. The cell suspension was dropped onto glass slides in a moist box and dried. The slides were checked under a phase-contrast microscope. For meiotic spread preparation, immature flower buds measuring approximately $0.5 \mathrm{~mm}$ in length were directly fixed in ethanol/acetic acid (3:1) for $24 \mathrm{~h}$ and stored in $70 \%$ ethanol. The anthers were isolated and used for spread preparation. The procedure was the same as that for mitotic spread preparation.
The selected slides with well-spread metaphase chromosomes or desired stage of mitotic process were UV-crosslinked for $2 \mathrm{~min}$. A probe solution containing in $2 \times \mathrm{SSC}$ and $1 \times \mathrm{TE}$ was then added on the slides. After denaturation in boiling water for $5 \mathrm{~min}$, the slides with probe were incubated at $55^{\circ} \mathrm{C}$ in a humid chamber for 8-12 h. The slides were then washed thrice in $2 \times$ SSC, with each washing lasting for $5 \mathrm{~min}$, at $50^{\circ} \mathrm{C}$ and finally counterstained with 4',6-diamidino-2-phenylindole (DAPI) (Vector Laboratories, Burlingame, USA). The FISH images were captured with an ANDOR CCD under an Olympus BX63 fluorescence microscope. The images were processed by Adobe Photoshop 7.0.

\section{Additional files}

Additional file 1: Annotation of full-length LTR-REs in spinach genome. (XLS 3011 kb)

Additional file 2: Topological layout and consensus sequence of satellite DNAs. (DOC $1441 \mathrm{~kb}$ )

Additional file 3: TE concensus sequences generated by RepeatModeler in spinach genome. (FASTA $1073 \mathrm{~kb}$ )

Additional file 4: TE dataset detected by signature-based methods in spinach genome. (FASTA $122852 \mathrm{~kb}$ )

Additional file 5: The primers used for amplification of the RT sequences of lineages in spinach. (DOC $42 \mathrm{~kb}$ )

\section{Abbreviations}

FISH: Fluorescence in situ hybridization; INT: Integrase; LTR: Long terminal repeat; MITE: Miniature inverted repeat transposable element; PBS: Primerbinding site; PPT: Polypurine tract; RE: Retrotransposon; RH: RNAseH; RT: Reverse transcriptase; TE: Transposable element; TIR: Terminal inverted repeat; TSD: Target site duplication

Acknowledgements

Not applicable.

\section{Funding}

This work was financially supported by grants from the National Natural Science foundation of China (31470334 and 31770346), Program for Innovative Research Team (in Science and Technology) in University of Henan Province (17IRTSTHN017), and the Foundation for Young Teachers in Colleges and Universities of Henan Province (2016GGJS-051).

\section{Availability of data and materials}

All the TE annotated results and the sequences are available as part of the Additional files. They are also deposited to Dryad (http://datadryad.org/) with DOI number: doi:https://doi.org/10.5061/dryad.4053d31.

\section{Authors' contributions}

SFL and WJG designed the study. SFL and JRL performed TE analysis. YJG and BXW performed FISH analysis. SFL and WJG wrote the manuscript. NL, DXZ, and CLD provided helpful suggestions in data analysis. All authors read and approved the paper.

Ethics approval and consent to participate Not applicable

Consent for publication

Not applicable

Competing interests

The authors declare that they have no competing interests. 


\section{Publisher's Note}

Springer Nature remains neutral with regard to jurisdictional claims in published maps and institutional affiliations.

\section{Author details}

'College of Life Sciences, Henan Normal University, Xinxiang 453007, China.

${ }^{2}$ College of Life Science, Shanxi Datong University, Datong 037009, China.

Received: 5 November 2018 Accepted: 7 January 2019

Published online: 18 January 2019

\section{References}

1. Schnable PS, Ware D, Fulton RS, Stein JC, Wei F, Pasternak S, et al. The B73 maize genome: complexity, diversity, and dynamics. Science. 2009;326:1112-5.

2. Kumar A, Bennetzen JB. Plant retrotransposons. Ann Rev Genet. 1999:33: 479-532.

3. Kalendar R, Flavell AJ, Ellis THN, Sjakste T, Moisy C, Schulman AH. Analysis of plant diversity with retrotransposon-based molecular markers. Heredity. 2011;106:520-30.

4. Sharma S, Raina SN. Organization and evolution of highly repeated satellite DNA sequences in plant chromosomes. Cytogenet Genome Res. 2005;109: $15-26$.

5. Bennetzen $J$, Wang $H$. The contributions of transposable elements to the structure, function, and evolution of plant genomes. Annu Rev Plant Biol. 2014;65:505-30

6. Harkess A, Mercati F, Abbate L, McKain M, Pires JC, Sala T, Sunseri F, Falavigna A, Leebens-Mack J. Retrotransposon proliferation coincident with the evolution of dioecy in Asparagus. G3. 2016;6:2679-85.

7. Li SF, Su T, Cheng GQ, Wang BX, Li X, Deng CL, Gao WJ. Chromosome evolution in connection with repetitive sequences and epigenetics in plant. Genes. 2017;8:290.

8. Martin A, Troadec C, Boualem A, Rajab M, Fernandez R, Morin H, Pitrat M, Dogimont C, Bendahmane A. A transposon-induced epigenetic change leads to sex determination in melon. Nature. 2009;461:1135-8.

9. Cho J, Paszkowski J. Regulation of rice root development by a retrotransposon acting as a microRNA sponge. elife. 2017;6:e30038.

10. Li SF, Zhang GJ, Yuan JH, Deng CL, Gao WJ. Repetitive sequences and epigenetic modification: inseparable partners play important roles in the evolution of plant sex chromosomes. Planta. 2016;243:1083-95.

11. Kejnovsky E, Hobza R, Cermak T, Kubat Z, Vyskot B. The role of repetitive DNA in structure and evolution of sex chromosomes in plants. Heredity. 2009;102:533-41.

12. lizuka M, Janick J. Cytogenetic analysis of sex determination in Spinacia oleracea. Genetics. 1962;47:1225-41.

13. Deng CL, Qin RY, Gao J, Cao Y, Li SF, Gao WJ, Lu LD. Identification of sex chromosome of spinach by physical mapping of $45 \mathrm{~S}$ rDNAs by FISH. Caryologia. 2012;65:322-7.

14. Xu C, Jiao C, Sun H, Cai X, Wang X, Ge C, et al. Draft genome of spinach and transcriptome diversity of 120 Spinacia accessions. Nat Commun. 2017; 8:15275.

15. Lerat E. Identifying repeats and transposable elements in sequenced genomes: how to find your way through the dense forest of programs. Heredity. 2010;104:520-33.

16. Bergman CM, Quesneville $H$. Discovering and detecting transposable elements in genome sequences. Brief Bioinform. 2007:8:382-92.

17. Barghini E, Natali L, Giordani T, Cossu RM, Scalabrin S, Cattonaro F, et al. LTR retrotransposon dynamics in the evolution of the olive (Olea europaea) genome. DNA Res. 2014;22:91.

18. Wicker T, Gundlach H, Spannagl M, Uauy C, Borrill P, Ramírez-González RH, et al. Impact of transposable elements on genome structure and evolution in bread wheat. Genome Biol. 2018:19:103.

19. Permal E, Flutre T, Quesneville H. Roadmap for annotation transposable elements in eukaryote genomes. Methods Mol Biol. 2012;859:53-68.

20. Platt RN 2nd, Blanco-Berdugo L, Ray DA. Accurate transposable element annotation is vital when analyzing new genome assemblies. Genome Biol Evol. 2016:8:403-10.

21. Cai J, Liu X, Vanneste K, Proost S, Tsai WC, Liu KW, et al. The genome sequence of the orchid Phalaenopsis equestris. Nat Genet. 2015;47:65-72.

22. Sessegolo C, Burlet N, Haudry A. Strong phylogenetic inertia on genome size and transposable element content among 26 species of flies. Biol Lett. 2016;12:20160407
23. Li F, Fan G, Wang K, Sun F, Yuan Y, Song G, et al. Genome sequence of the cultivated cotton Gossypium arboreum. Nat Genet. 2014;46:567-72.

24. Singh R, Ong-Abdullah M, Low ETL, Manaf MAA, Rosli R, Nookiah R, et al. Oil palm genome sequence reveals divergence of interfertile species in old and new worlds. Nature. 2013;500:335-9.

25. Paz RC, Kozaczek ME, Rosli HG, Andino NP, Sanchez-Puerta MV. Diversity, distribution and dynamics of full-length Copia and Gypsy LTR retroelements in Solanum lycopersicum. Genetica. 2017;6:1-14.

26. Sanmiguel $P$, Bennetzen JL. Evidence that a recent increase in maize genome size was caused by the massive amplification of intergene retrotransposons. Ann Bot. 1998;82:37-44.

27. Neumann P, Kobližková A, Navrátilová A, Macas J. Significant expansion of Vicia pannonica gnome size mediated by amplification of a single type of giant retroelement. Genetics. 2006;173:1047-56.

28. Hirochika $\mathrm{H}$, Okamoto $\mathrm{H}$, Kakutani $\mathrm{T}$. Silencing of retrotransposons in Arabidopsis and reactivation by the $d d m 1$ mutation. Plant Cell. 2000;12: 357-68.

29. Paz RC, Rendina González AP, Ferrer MS, Masuelli RM. Short-term hybridization activates Tnt1 and Tto1 Copia retrotransposons in wild tuberbearing Solanum species. Plant Biol. 2015;17:860-9.

30. Oliver KR, Greene WK. Transposable elements: powerful facilitators of evolution. BioEssays. 2009;31:703-14.

31. Lisch D. Epigenetic regulation of transposable elements in plants. Annu Rev Plant Biol. 2009;60:43-66

32. Ma J, Bennetzen JL. Rapid recent growth and divergence of rice nuclear genomes. Proc Natl Acad Sci U S A. 2004;101:12404-10.

33. Charles M, Belcram H, Just J, Huneau C, Viollet A, Couloux A, et al. Dynamics and differential proliferation of transposable element during the evolution of the B and A genomes of wheat. Genetics. 2008;180:1071-86.

34. Erlandsson R, Wilson JF, Paabo S. Sex chromosomal transposable element accumulation and male-driven subtitutional evolution in humans. Mol Bio Evol. 2000;17:804-12.

35. VanBuren R, Ming R. Dynamic transposable element accumulation in the nascent sex chromosomes of papaya. Mob Genet Element. 2013;3:e23462.

36. VanBuren R, Zeng F, Chen C, Zhang J, Wai CM, Han J, et al. Origin and domestication of papaya $Y^{h}$ chromosome. Genome Res. 2015:25:524-33.

37. Mariotti B, Manzano S, Kejnovsky E, Vyskot B, Jamilena M. Accumulation of Y-specific satellite DNAs during the evolution of Rumex acetosa sex chromosomes. Mol Genet Genomics. 2009;281:249-59.

38. Cermak T, Kubat Z, Hobza R, Koblizkova A, Widmer A, Macas J, Vyskot B, Kejnovsky $E$. Survey of repetitive sequence in Silene latifolia with respect to their distribution on sex chromosome. Chromosom Res. 2008;16:961-76.

39. Kubat Z, Zluvova J, Vogel I, Kovacova V, Cermak T, Cegan R, Hobza R, Vyskot B, Kejnovsky E. Possible mechanisms repsonsible for absence of a retrotransposon family on a plant Y chromosome. New Phytol. 2014; 202:662-78

40. Steflova P, Tokan V, Vogel I, Lexa M, Macas J, Novak P, Hobza R, Vyskot B, Kejnovsky E. Contrasting patterns of transposable element and satellite distribution on sex chromosomes $\left(X Y_{1} Y_{2}\right)$ in the dioecious plant Rumex acetosa. Genome Biol Evol. 2013;5:769-82

41. Ming R, Bendahmane A, Renner SS. Sex chromosomes in land plants. Annu Rev Plant Biol. 2011;62:485-514.

42. Charlesworth D. Plant contributions to our understanding of sex chromosome evolution. New Phytol. 2015;208:52-65.

43. Negrutiu I, Vyskot B, Barbacar N, Georgiev S, Moneger F. Dioecious plants. A key to the early events of sex chromosome evolution. Plant Physiol. 2001; 127:14218-24

44. Wang JP, Na JK, Yu QY, Gschwend AR, Han J, Zeng F, et al. Sequencing papaya $X$ and $Y^{h}$ chromosomes reveals molecular basis of incipient seX chromosome evolution. Proc Natl Acad Sci U S A. 2012:109:13710-5.

45. Holstein N, Renner SS. Niche conservation? Biome switching within and between species of the African genus Coccinia (Cucurbitaceae). BMC Evol Biol. 2011;11:28

46. Yu Q, Navajas-Pérez $R$, Tong E, Robertson J, Moore PH, Paterson AH, Ming R. Recent origin of dioecious and gynodioecious $Y$ chromosomes in papaya. Trop Plant Biol. 2008;1:49-57.

47. Spigler RB, Lewers KS, Main DS, Ashman TL. Genetic mapping of sex determination in a wild strawberry, Fragaria virginiana, reveals earliest form of sex chromosome. Heredity. 2008;101:507-17.

48. del Bosque ME, Navajas-Pérez R, Panero JL, Fernández-González A, GarridoRamos MA. A satellite DNA evolutionary analysis in the North American 
endemic dioecious plant Rumex hastatulus (Polygonaceae). Genome. 2011; 54:253-60.

49. Vitte C, Estep MC, Leebens-Mack J, Bennetzen JL. Young, intact and nested retrotransposons are abundant in the onion and asparagus genomes. Ann Bot. 2013;112:881-9.

50. Du J, Tian Z, Hans CS, Laten HM, Cannon SB. Evolutionary conservation, diversity and specificity of LTR-retrotransposons in flowering plants: insights from genome-wide analysis and multi-specific comparison. Plant J. 2010;63: 584-98.

51. Ellinghaus D, Kurtz S, Willhoeft U. LTRharvest, an efficient and flexible software for de novo detection of LTR retrotransposons. BMC Bioinformatics. 2008;9:18.

52. Steinbiss S, Willhoeft U, Gremme G, Kurtz S. Fine-grained annotation and classification of de novo predicted LTR retrotransposons. Nucleic Acids Res. 2009:37:7002-13.

53. Lowe TM, Eddy SR. tRNAscan-SE: a program for improved detection of transfer RNA genes in genomic sequence. Nucleic Acids Res. 1997;25: 955-64

54. Llorens C, Futami R, Covelli L, et al. The Gypsy Database (GyDB) of mobile genetic elements: release 2.0. Nucleic Acids Res. 2011;39:D70-4.

55. Punta M, Coggill PC, Eberhardt RY, Mistry J, Tate J, Boursnell C, et al. The Pfam protein families database. Nucleic Acids Res. 2012:40:D290-301.

56. Novák P, Neumann P, Pech J, Steinhaisl J, Macas J. RepeatExplorer: a galaxy-based web server for genome-wide characterization of eukaryotic repetitive elements from next-generation sequence reads. Bioinformatics. 2013:29:792-3.

57. Rho M, Tang H. MGEScan-non-LTR: computational identification and classification of autonomous non-LTR retrotransposons in eukaryotic genomes. Nucleic Acids Res. 2009;37:e143.

58. Xiong W, He L, Lai J, Dooner HK, Du C. HelitronScanner uncovers a large overlooked cache of Helitron transposons in many plant genome. Proc Natl Acad Sci U S A. 2014;111:10263-8.

59. Ge R, Mai G, Zhang R, Wu X, Wu Q, Zhou F. MUSTv2: an improved de novo detection program for recently active miniature inverted repeat transposable elements (MITEs). J Integr Bioinform. 2017;14:20170029.

60. Han Y, Wessler SR. MITE-hunter: a program for discovering miniature inverted-repeat transposable elements from genomic sequences. Nucleic Acids Res. 2010;38:e199.

61. Li W, Godzik A. CD-HIT: a fast program for clustering and comparing large sets of protein or nucleotide sequences. Bioinformatics. 2006;22:1658-9.

62. Edgar RC. MUSCLE: multiple sequence alignment with high accuracy and high throughput. Nucleic Acids Res. 2004;32:1792-7.

63. Price MN, Dehal PS, Arkin AP. FastTree2 - approximately maximumlikelihood trees for large alignments. PLoS One. 2010;5:e9490.

64. SanMiguel P, Gaut BS, Tikhonov A, Nakajima Y, Bennetzen UL. The paleontology of intergene retrotransposons of maize. Nat Genet. 1998;20:43-5.

65. Rice P, Longden I, Bleasby A. EMBOSS: the European molecular biology open software suite. Trends Genet. 2000;16:276-7.

66. Kimura M. A simple method for estimating evolutionary rates of base substitutions through comparative studies of nucleotide sequences. J Mol Evol. 1980;16:111-20

67. Göl S, Göktay M, Allmer J, Doğanlar S, Frary A. Newly developed SSR markers reveal genetic diversity and geographical clustering in spinach (Spinacia oleracea). Mol Gen Genomics. 2017;292:847-55.

68. Yang X, Liu D, Wu J, Zou J, Xiao X, Zhao F, Zhu B. HTQC: a fast quality control toolkit for Illumina sequencing data. BMC Bioinformatics. 2013;14:33.

69. Novák P, Robledillo LÁ, Kobližková A, Vrbová I, Neumann P, Macas J. TAREAN: a computational tool for identification and characterization of satellite DNA from unassembled short reads. Nucleic Acids Res. 2017:45:e111.

70. Crooks GE, Hon G, Chandonia JM, Brenner SE. WebLogo: a sequence logo generator. Genome Res. 2004:14:1188-90.

71. Birchler JA, Albert PS, Gao Z. Stability of repeated sequence clusters in hybrids of maize as revealed by FISH. Trop Plant Biol. 2008;1:34-9.

Ready to submit your research? Choose BMC and benefit from:

- fast, convenient online submission

- thorough peer review by experienced researchers in your field

- rapid publication on acceptance

- support for research data, including large and complex data types

- gold Open Access which fosters wider collaboration and increased citations

- maximum visibility for your research: over $100 \mathrm{M}$ website views per year

At BMC, research is always in progress.

Learn more biomedcentral.com/submissions 